\title{
The effects of insulin and liraglutide on osteoprotegerin and vascular calcification in vitro and in patients with type 2 diabetes
}

\author{
Colin Davenport, Wan A Mahmood', Hannah Forde, David T Ashley, Amar Agha, \\ John McDermott' ${ }^{1}$, Seamus Sreenan ${ }^{1}$, Christopher J Thompson, Frank McGrath', \\ Brendan McAdam ${ }^{3}$, Philip M Cummins ${ }^{4}$ and Diarmuid Smith
}

Department of Academic Endocrinology, Diabetes Day Centre, Beaumont Hospital, County Dublin, Dublin 9, Ireland, 'Department of Diabetes and Endocrinology, Connolly Hospital, Blanchardstown, County Dublin, Dublin 9, Ireland, Departments of ${ }^{2}$ Radiology and ${ }^{3}$ Cardiology, Beaumont Hospital, County Dublin, Dublin 9, Ireland and ${ }^{4}$ School of Biotechnology, Centre for Preventive Medicine, Dublin City University, County Dublin, Dublin 9, Ireland
Correspondence should be addressed to C Davenport

Email

drcdavenport@gmail.com

\begin{abstract}
Objective: Vascular calcification (VC) is inhibited by the glycoprotein osteoprotegerin (OPG). It is unclear whether treatments for type 2 diabetes are capable of promoting or inhibiting VC. The present study examined the effects of insulin and liraglutide on i) the production of OPG and ii) the emergence of VC, both in vitro in human aortic smooth muscle cells (HASMCs) and in vivo in type 2 diabetes.

Design/methods: HASMCs were exposed to insulin glargine or liraglutide, after which OPG production, alkaline phosphatase $(A L P)$ activity and levels of Runx2, ALP and bone sialoprotein (BSP) mRNA were measured. A prospective, nonrandomised human subject study was also conducted, in which OPG levels and coronary artery calcification (CAC) were measured in a type 2 diabetes population before and 16 months after the commencement of either insulin or liraglutide treatment and in a control group that took oral hypoglycemics only.

Results: Exposure to insulin glargine, but not liraglutide, was associated with significantly decreased OPG production (11 $913 \pm 1409 \mathrm{pg} / 10^{4}$ cells vs $282 \pm 13 \mathrm{pg} / 10^{4}$ cells, control vs $10 \mathrm{nmol} / \mathrm{l}$ insulin, $\left.P<0.0001\right)$, increased $A L P$ activity $(0.82 \pm 0.06$ $\mathrm{IU} / 10^{4}$ cells vs $2.40 \pm 0.16 \mathrm{IU} / 10^{4}$ cells, control vs $10 \mathrm{nmol} / \mathrm{l}$ insulin, $P<0.0001$ ) and increased osteogenic gene expression by HASMCs. In the clinical study $(n=101)$, insulin treatment was associated with a significant reduction in OPG levels and, despite not achieving full statistical significance, a trend towards increased CAC in patients.

Conclusion: Exogenous insulin down-regulated OPG in vitro and in vivo and promoted VC in vitro. Although neither insulin nor liraglutide significantly affected CAC in the present pilot study, these data support the establishment of randomised trials to investigate medications and VC in diabetes.
\end{abstract}

\section{Introduction}

Vascular calcification (VC) has been shown to exert a number of adverse effects on the cardiovascular (CV) system, and it is particularly prevalent in patients with type 2 diabetes mellitus, who tend to develop VC to a greater degree and at an earlier age $(1,2,3)$. At the cellular level, VC is the end product of a complex pathophysiological process in which cell populations such as vascular smooth muscle cells (VSMC) display osteoblastic activity similar to that seen in skeletal bone formation $(1,4)$. The glycoprotein osteoprotegerin (OPG), which is produced by VSMCs, has been identified as an inhibitor of this process $(5,6)$. Short-term clamp studies in human subjects have suggested that insulin decreases the production of OPG, while in rat models, glucagon-like (c) 2015 European Society of Endocrinology Printed in Great Britain
Published by Bioscientifica Ltd 
peptide-1 (GLP-1) has been reported to increase OPG production $(7,8)$. These data, which have yet to be confirmed in long-term studies in human subjects, nevertheless raise the possibility that treatment with insulin or GLP-1 analogues may promote or inhibit VC respectively in type 2 diabetes. In keeping with this hypothesis, a small number of in vitro studies have reported a promotion of osteoblastic activity in VSMCs following exposure to insulin $(9,10,11,12)$. These findings, however, have been limited by experimental protocols that utilise extremely high concentrations of human insulin, the measurement of suboptimal endpoints of osteoblastic activity (e.g. calcium deposition) and the use of nonhuman vascular cell populations. Furthermore, the effects of insulin analogues, which in comparison to endogenous insulin have different chemical structures and domains of biological activity, were not assessed, and the effects of GLP-1 analogues on osteoblastic activity in VSMCs have not been studied under any setting.

The present study was designed to expand on the limited evidence base in this area by examining the effects of insulin and GLP-1 analogue therapy on OPG production by human aortic smooth muscle cells (HASMCs), along with the chronic effects of these medications on circulating OPG concentrations in patients with type 2 diabetes. At the same time, we also measured the effects of these therapies on osteoblastic behaviour by HASMCs, along with their effects on coronary artery calcification (CAC) in this patient population.

\section{Subjects and methods}

\section{HASMC culture and maintenance}

Differentiated (positive for $\alpha$-actin) HASMCs were used between passages 5 and 9. Smooth muscle cell growth medium 2 (additives included $0.05 \mathrm{ml} / \mathrm{ml} \mathrm{FCS,} 0.5 \mathrm{ng} / \mathrm{ml}$ epidermal growth factor and $2 \mathrm{ng} / \mathrm{ml}$ basic fibroblast growth factor, with insulin omitted from the media mix) was obtained from Promocell GmbH (Heidelberg, Germany). Penicillin (100 IU/ml) and streptomycin $(100 \mu \mathrm{g} / \mathrm{ml})$ were added to the media mix. While growing, HASMCs were maintained in a humidified incubator at $37^{\circ} \mathrm{C}$ and $5 \% \mathrm{CO}_{2}$.

\section{Exposure of HASMCs to experimental conditions}

Experiments were conducted in standard six-well culture dishes, and they commenced once the HASMC populations had achieved confluency. Experiments were performed in triplicate, with a final $n$ for each experimental condition of 18 . For the experiments, HASMCs were exposed either to insulin glargine (Sanofi-Aventis, Surrey, $\mathrm{UK}$ ), which was added to the media at $1 \mathrm{nmol} / \mathrm{l}$ (pathophysiological concentrations observed in insulin-resistant patient populations) and $10 \mathrm{nmol} / 1$ concentrations (supraphysiological) or to liraglutide (Novo Nordisk, Bagsvaerd, Denmark) at $30 \mathrm{nmol} / \mathrm{l}$ (levels normally achieved during liraglutide treatment) and $300 \mathrm{nmol} / 1$ concentrations (supraphysiological) $(13,14)$. HASMCs cultured in media without the addition of either insulin glargine or liraglutide represented the control conditions. HASMCs were exposed to experimental conditions for $72 \mathrm{~h}$.

\section{Measurement of OPG production and osteoblastic activity by HASMCs}

Following $72 \mathrm{~h}$ of exposure, we harvested the supernatant media surrounding the HASMCs along with the HASMC mRNA (mRNA harvested with Trizol, Invitrogen). Samples were stored at $-80^{\circ} \mathrm{C}$, and the relevant assays were performed within 1 month as part of a single freeze-thaw cycle. Alkaline phosphatase $(A L P)$ activity, a critical marker of osteoblastic activity, was measured in the supernatant media via the Qunatichrom kit (BioAssay Systems, Hayward, CA, USA), as were concentrations of OPG via ELISA (OPG Duoset kit, R\&D Systems, Minneapolis, MN, USA). The intra-assay coefficient of variation (CV) for both assays was $<5 \%$, as was the inter-assay $\mathrm{CV}$ for the Quantichrom kit. mRNA samples were reverse transcribed into cDNA to allow for the measurement of HASMC osteogenic gene expression by quantitative RT-PCR (Applied Biosystems 7900 HT Fast RT-PCR System). S18 was employed as a housekeeping gene to normalise the cDNA quantity between samples. Primer sequences for $S 18$ and the osteogenic differentiation genes runt-related transcription factor 2 (Runx2), ALP and bone sialoprotein $(B S P)$ are listed in Table 1 . HASMC number and viability were routinely measured for seeding density purposes using the advanced detection and accurate measurement cell counter (Digital Bio, Seoul, South Korea).

\section{Clinical study design and protocol}

The clinical component of the present research was conducted in three hospitals in the Dublin area Beaumont Hospital, Connolly Hospital and the Sports Surgery Clinic - between January 2011 and November 2013. The study was approved by the local ethics committees and was performed in accordance with the 
Table 1 Sequences for S18, Runx2, BSP and ALP primer sets.

\begin{tabular}{|c|c|c|c|}
\hline Gene & Primer sequence & $\begin{array}{c}\text { Annealing } \\
\text { temperature }\left({ }^{\circ} \mathrm{C}\right)\end{array}$ & $\begin{array}{l}\text { Product } \\
\text { size }(b p)\end{array}$ \\
\hline S18 & $\begin{array}{l}\text { Forward: 5'-CAGCCACCCGAGATTGAGCA-3' } \\
\text { Reverse: } 5^{\prime} \text {-TAGTAGCGACGGGCGGTGTG-3' }\end{array}$ & 62 & 250 \\
\hline Runx2 & $\begin{array}{l}\text { Forward: 5'-GGTACCAGATGGGACTGTGG-3' } \\
\text { Reverse: } 5^{\prime} \text {-GAGGCGGTCAGAGAACAAAC-3' }\end{array}$ & 59 & 315 \\
\hline$B S P$ & $\begin{array}{l}\text { Forward: 5'- TCAGCATTTTTGGGAATGGCC-3' } \\
\text { Reverse: } 5^{\prime} \text { - GAGGTTGTTGTCTTCGAGGT-3' }\end{array}$ & 61 & 567 \\
\hline$A L P$ & $\begin{array}{l}\text { Forward: 5'- GCCTGGCTACAAGGTGGTG-3' } \\
\text { Reverse: } 5^{\prime}-\text { GGCCAGAGCGAGCAGC-3' }\end{array}$ & 58 & 293 \\
\hline
\end{tabular}

Helsinki Declaration. Because the present study was the first to provide clinical data on the issue in question, a nonrandomised, observational design was chosen for this multicentre prospective research.

\section{Patient recruitment}

Three groups of patients with type 2 diabetes were recruited. The first group was the control group, and it consisted of patients taking metformin \pm sulphonylurea for the duration of the study. The second and third groups consisted of patients who required optimisation of their diabetes control and were started on either liraglutide or a basal-bolus insulin regime in addition to their metformin \pm sulphonylurea treatment. Patients in the control group were recruited from the diabetes outpatient clinics, whereas patients in the liraglutide and insulin groups were recruited opportunistically when they were referred by their diabetes team to a diabetes day centre to commence either liraglutide or insulin. Exclusion criteria included childbearing potential, a stroke or myocardial infarction in the preceding 6 months, an estimated glomerular filtration rate (eGFR) of $<60 \mathrm{ml} / \mathrm{min}$ per $1.73 \mathrm{~m}^{2}$, active malignancy and treatment with medications known to interfere with circulating OPG concentrations and/or to alter bone metabolism (including denosumab, bisphosphonates, thiazolideinediones, dipeptidyl peptidase- 4 inhibitors, strontium or calcitonin) within the preceding 6 months. If patients required treatment with such medications, or if they ceased taking their liraglutide or insulin during the time frame of the study, their participation in the study ended and they were not included in the final analysis. Medication compliance was assessed via 6 monthly telephone interviews during the study time frame.

\section{Measurement of clinical endpoints}

Immediately before the commencement of either liraglutide or insulin, and again after 16 months of exposure to these medications, anthropometric measurements, fasting serum samples and CAC were measured. Circulating OPG concentrations were measured with the Duoset ELISA assay, whereas lipids, serum creatinine and $\mathrm{HbA1c}$ were measured using standard laboratory techniques. CAC burden was measured via computed tomography (CT) using the Agatson scoring system with a GE Lightspeed VCT 64 slice CT scanner (GE Healthcare, Milwaukee, WI, USA) (15). CAC burden was calculated by a single radiologist who was blinded to treatment status.

\section{Statistical analysis}

Data from the in vitro experiments were analysed using GraphPad Prism version 4.03 (GraphPad Software, La Jolla, CA, USA) and are expressed in means \pm s.e.M. (normality was tested for via the Kolmogorov-Smirnov test). Student's unpaired $t$-test was employed for pair-wise comparisons. Data from the clinical study were analysed using SPSS version 16 (SPSS Incorporated). Data are reported as means \pm s.E.M. or median (25th-75th percentile), as appropriate. Differences in study variables from baseline to study completion were analysed using a paired $t$-test or a Wilcoxon signed-rank test, as appropriate. To test whether exposure to liraglutide or insulin influenced the observed changes in either OPG or CAC over the study duration, an ANCOVA was performed, and it included as its covariates the values which differed between the three groups at baseline. The covariates that were added, therefore, included the pre-intervention OPG or CAC values as well as those variables noted as being significantly different between the groups in Table 2. After adjustment for pre-intervention covariates in this fashion, the ANCOVA was performed to identify any statistically significant differences in OPG/CAC concentrations between the groups post-intervention. A post hoc analysis using the Bonferroni adjustment was then performed to correct for the multiple comparisons performed during this analysis. 
Table 2 Characteristics of clinical study subjects at baseline. Data are given as means \pm s.E.M. or median (25th-75th percentile), as appropriate.

\begin{tabular}{l}
\hline Variable \\
\hline Gender (male/female; \%) \\
Age (years) \\
Metabolic characteristics \\
Weight $(\mathrm{kg})$ \\
BMI (kg/m²) \\
HbA1c (mmol/mol) \\
HbA1c (\%) \\
Total cholesterol (mmol/l) \\
LDL cholesterol (mmol/l) \\
HDL cholesterol (mmol/l) \\
Triglycerides (mmol/l) \\
eGFR (ml/min per 1.73 $\mathrm{m}^{2}$ ) \\
Calcification data \\
OPG (pg/ml) \\
Calcium (mmol/l) \\
CAC score \\
Medical history \\
Years since diabetes dx \\
Smoking (yes/no; \%) \\
IHD (yes/no; \%) \\
CVA (yes/no; \%) \\
Statins (yes/no; \%) \\
ACEi/ARB (yes/no; \%) \\
Sulphonylureas (yes/no; \%)
\end{tabular}

\begin{tabular}{c}
\hline Metformin \\
$35 / 9 ; 79.5 \%$ \\
$63.8 \pm 1.3 * *$ \\
\\
$89.2(75.5-96.2)^{* *}$ \\
$30.1(27.5-33.2)^{* *}$ \\
$49(46-53)^{* *}$ \\
$6.6(6.4-7)^{* *}$ \\
$3.8 \pm 0.1$ \\
$1.9 \pm 0.1$ \\
$1.16(1.00-1.34)$ \\
$1.44(1.18-2.38)$ \\
$98 \pm 3 *$ \\
$1080 \pm 58$ \\
$2.36 \pm 0.01$ \\
$219(3-768)$ \\
\\
$4.0(3.0-6.0)$ \\
$9 / 35 ; 20.5 \%$ \\
$3 / 41 ; 6.8 \%$ \\
$1 / 43 ; 2.3 \%$ \\
$37 / 7 ; 84.1 \%$ \\
$30 / 14 ; 68.2 \%$ \\
$4 / 40 ; 9.1 \% *$ \\
\end{tabular}

\begin{tabular}{c}
\hline Liraglutide \\
\hline $21 / 11 ; 65.6 \%$ \\
$53.6 \pm 1.7$ \\
$109.8(100.0-121.1)^{\dagger}$ \\
$36.8(33.3-41.1)$ \\
$58(52-66)^{\dagger \dagger}$ \\
$7.5(6.9-8.2)^{\dagger \dagger}$ \\
$4.1 \pm 0.2$ \\
$2.0 \pm 0.1$ \\
$1.11(0.95-1.29)$ \\
$1.55(1.21-2.33)$ \\
$110 \pm 4$ \\
$1192 \pm 77$ \\
$2.38 \pm 0.02$ \\
$5(0-150)$ \\
\\
$5.0(3.0-6.5)$ \\
$6 / 26 ; 17.1 \%$ \\
$0 / 32 ; 0 \%$ \\
$0 / 32 ; 0 \%$ \\
$27 / 5 ; 84.4 \%$ \\
$23 / 9 ; 71.9 \%$ \\
$11 / 21 ; 34.4 \%$ \\
\end{tabular}

\begin{tabular}{c}
\hline Insulin \\
\hline $15 / 10 ; 60.0 \%$ \\
$59.2 \pm 2.3$
\end{tabular}

${ }^{*}$ For comparison between control and liraglutide groups, ${ }^{*} P<0.05, * * P<0.0005 .{ }^{\dagger}$ For comparison between liraglutide and insulin groups, ${ }^{\dagger} P<0.05$, ${ }^{+\dagger} P<0.005$. ${ }^{\ddagger}$ For comparison between insulin and control groups, ${ }^{\ddagger} P<0.05,{ }^{\ddagger \neq} P<0.005$. ACEi, angiotensin converting enzyme inhibitor; $A R B$, angiotensin receptor blocker; CVA, cerebrovascular accident; dx, diagnosis; IHD, ischemic heart disease; NS, non-significant.

\section{Results}

\section{Exposure to insulin glargine, but not liraglutide, decreased the secretion of OPG from HASMCs}

OPG was noted to be secreted by HASMCs under basal conditions (mean \pm s.e.m. $11913 \pm 1409$ pg/10 000 cells), but following exposure to both 1 and $10 \mathrm{nmol} / 1$ insulin glargine (pathophysiological and supraphysiological concentrations respectively), the secretion of OPG decreased by $>95 \%$ to $201 \pm 11$ and $282 \pm 13 \mathrm{pg} / 10000$ cells respectively $(P<0.0001$ for both changes). In contrast, liraglutide had no significant effect on OPG secretion by HASMCs $(9743 \pm 521 \mathrm{pg} / 10000$ cells under basal conditions vs $10081 \pm 762 \mathrm{pg} / 10000$ cells and $7693 \pm$ $2346 \mathrm{pg} / 10000$ cells for 30 and $300 \mathrm{nmol} / \mathrm{l}$ liraglutide respectively).

\section{Exposure to insulin glargine, but not liraglutide, increased osteoblastic activity in HASMCs}

A significant increase in $A L P$ activity was noted in the supernatant media surrounding the cells following exposure to insulin glargine (Fig. 1A), but no change in
$A L P$ activity was observed following exposure to liraglutide at either concentration (Fig. 1B). When osteogenic gene expression was assessed via analysis of Runx2, ALP and BSP mRNA levels, pro-osteogenic changes were observed following exposure to insulin glargine, with increased expression of both Runx 2 and $A L P$ at pathophysiological and supraphysiological concentrations and increased expression of $B S P$ at supraphysiological concentrations (Fig. 2A). All of the increases were insulin dosedependent. No changes in osteogenic gene expression were noted following exposure to liraglutide (Fig. 2B).

\section{Baseline characteristics of the clinical population}

In the clinical study, 110 patients were recruited, but nine were excluded from analysis because of changes in medication during the study time frame $(n=7)$ or because of failure to attend for the final visit $(n=2)$. Data from 101 patients, therefore, were included in the final analysis, and their baseline characteristics are presented in Table 2 . The mean age of the entire study population was $59.4 \pm 10.1$ years. The control group comprised 44 patients on metformin \pm sulphonylurea therapy for the duration of 

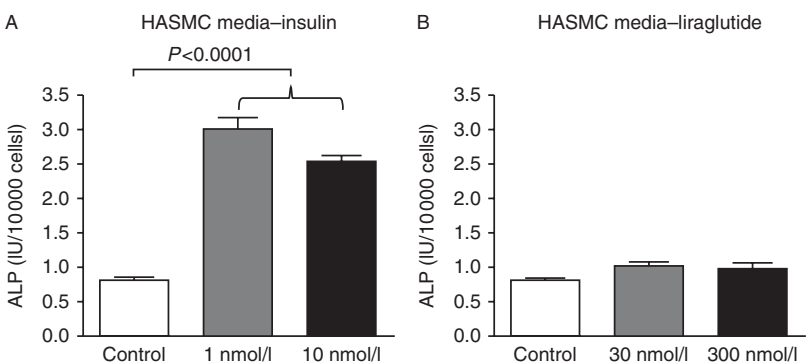

\section{Figure 1}

$A L P$ activity as measured by Quantichrom assay in supernatant media from cultures of HASMCs exposed to insulin glargine

(1 and $10 \mathrm{nmol} / \mathrm{l}$ ) (A) and liraglutide (30 and $300 \mathrm{nmol} / \mathrm{l})$ (B). ALP activity is displayed in IU per 10000 cells following correction for viable HASMC population. Data are given as means \pm s.E.M. $n=18$ for all experiments.

the study, whereas 32 patients commenced and remained on liraglutide (liraglutide group, mean dose \pm s.D. $1.72 \pm$ $0.19 \mathrm{mg}$ ) and 25 patients commenced and remained on insulin (insulin group, mean dose \pm s.D. 32.12 \pm 19.44 IU). Within the insulin group, 22 patients received insulin glargine and three received insulin detemir, and bolus insulin (insulin aspart) was added at mealtimes to the basal insulin in five of the patients. Reflecting the observational nature of the study, baseline differences in a number of variables existed between the groups, including a higher BMI in the liraglutide group and higher HbA1c and duration of diabetes in the insulin group (Table 2). No significant correlations were noted between CAC scores at baseline and OPG concentrations, duration of diabetes or HbA1c.

\section{Exposure to insulin therapy, but not liraglutide therapy, was associated with a decrease in circulating OPG concentrations}

The changes in baseline variables over the 16 months of the study are detailed in Table 3 . With regards to OPG, circulating OPG concentrations increased in the control group, decreased in the insulin group and did not change in the liraglutide group (Fig. 3). With regards to the presence of a treatment effect (i.e. whether exposure to insulin therapy accounted for the observed decrease in OPG), ANCOVA analysis indicated that exposure to insulin analogues was associated with a significant decrease in OPG $(P<0.005)$, and this relationship was observed to persist when correcting for all baseline differences between the groups, including age, eGFR, duration of diabetes, body weight, triglyceride levels, sulphonylurea use and HbA1c $(P<0.05)$.

\section{CAC increased in the group exposed to insulin but did not change in the group exposed to liraglutide}

With regards to $\mathrm{VC}, \mathrm{CAC}$ burden increased in the group exposed to insulin (Fig. 4), but it did not change alter significantly in the group exposed to liraglutide or in the control group (Table 3). Upon initial ANCOVA analysis, exposure to insulin analogues was associated with a significant increase in CAC $(P<0.0005$ for a treatment effect from insulin on $\mathrm{CAC}$ ). This relationship persisted when correcting for the baseline differences within the groups in terms of age, eGFR, duration of diabetes, body weight, triglyceride level and sulphonylurea use $(P<0.05$ for a treatment effect from insulin on CAC). Significance was lost, however, when the analysis was corrected for the baseline differences in HbA1c $(P=0.11$ for a treatment
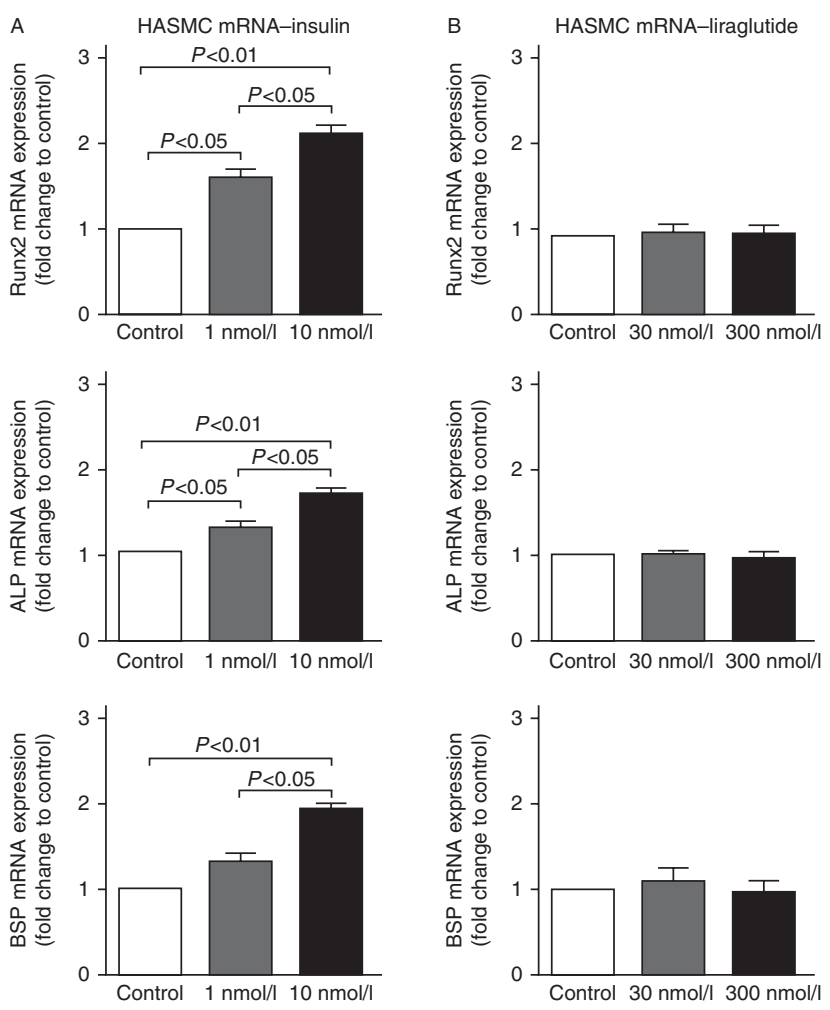

\section{Figure 2}

Effects of $72 \mathrm{~h}$ of exposure to insulin glargine ( 1 and $10 \mathrm{nmol} / \mathrm{l})$ (A) and liraglutide (at 30 and $300 \mathrm{nmol} / \mathrm{I}$ ) (B) on levels of Runx2, $A L P$ and $B S P$ mRNA from HASMCs. mRNA differences are expressed as the fold change in mRNA levels relative to control. Data are given as means \pm S.E.M. $n=18$ for all experiments. 
Table 3 Change in baseline variables over the duration of the study. Data are given as means \pm S.E.M. or median (25th-75th percentile), as appropriate.

\begin{tabular}{l} 
Variable \\
\hline Metabolic characteristics \\
Weight $(\mathrm{kg})$ \\
BMI $\left(\mathrm{kg} / \mathrm{m}^{2}\right)$ \\
HbA1c $(\mathrm{mmol} / \mathrm{mol})$ \\
HbA1c $(\%)$ \\
Total cholesterol $(\mathrm{mmol} / \mathrm{l})$ \\
LDL cholesterol $(\mathrm{mmol} / \mathrm{l})$ \\
HDL cholesterol $(\mathrm{mmol} / \mathrm{l})$ \\
Triglycerides $(\mathrm{mmol} / \mathrm{l})$ \\
eGFR (ml/min per $\left.1.73 \mathrm{~m}^{2}\right)$ \\
Calcification data \\
OPG $(\mathrm{pg} / \mathrm{ml})$ \\
Calcium $(\mathrm{mmol} / \mathrm{l})$ \\
CAC score
\end{tabular}

\begin{tabular}{c}
\hline Control \\
$-0.31 \pm 0.8$ \\
$-0.2 \pm 0.3$ \\
$-0.8 \pm 1.0$ \\
$-0.07 \pm 0.09$ \\
$-0.01 \pm 0.09$ \\
$-0.05(-0.41-0.14)$ \\
$-0.04(-0.13-0.11)$ \\
$0.07(-0.23-0.32)$ \\
$-0.9 \pm 1.6$ \\
$161 \pm 46^{*}$ \\
$0.01 \pm 0.02$ \\
$4(-21-66)$
\end{tabular}

\begin{tabular}{c}
\hline Liraglutide \\
$-2.9 \pm 1.0^{\dagger}$ \\
$-1.1 \pm 0.4^{\dagger}$ \\
$-7.7 \pm 1.5^{+\dagger}$ \\
$-0.7 \pm 0.14^{+\dagger}$ \\
$-0.07 \pm 014$ \\
$-0.30(-0.52-0.13)$ \\
$-0.02(-0.07-0.07)$ \\
$0.08(-0.32-0.71)$ \\
$2.4 \pm 0.02^{+\dagger}$ \\
$-75 \pm 66$ \\
$-0.01 \pm 0.02$ \\
$0(-8-4)$
\end{tabular}

\begin{tabular}{c}
\hline Insulin \\
$0.2 \pm 0.7$ \\
$0.2 \pm 0.3$ \\
$-5.3 \pm 3.2$ \\
$-0.48 \pm 0.29$ \\
$-0.23 \pm 0.2$ \\
$-0.22(-0.60-0.17)$ \\
$0(-0.23-0.07)$ \\
$-0.07(-0.53-0.49)$ \\
$-5.2 \pm 2.6$ \\
$-286 \pm 55^{\ddagger \neq}$ \\
$-0.01 \pm 0.02$ \\
$65(2-309)^{\ddagger \neq}$
\end{tabular}

${ }^{*}$ For comparison of change within the control group, ${ }^{*} P<0.005 .{ }^{\dagger}$ For comparison of change within the liraglutide group, ${ }^{\dagger} P<0.05,{ }^{\dagger \dagger} P<0.005,{ }^{\dagger+\dagger} P<0.0005$ ${ }^{\ddagger}$ For comparison of change within the insulin group, ${ }^{\ddagger} P<0.005,{ }^{\ddagger \ddagger} P<0.0005$.

effect). Thus, it was not possible to exclude baseline differences as a potential reason for the increased progression of CAC in the group exposed to insulin.

\section{Discussion}

The present study, which incorporated both laboratory and clinical research, is notable for providing the first clinical data on the effects of exogenous insulin and liraglutide on OPG levels and VC activity in type 2 diabetes. Furthermore, the study provides the first data on liraglutide and osteoblastic activity in vascular cells of any species and the first data on insulin glargine and osteoblastic activity in human vascular cells. Across both aspects of the present study, in vitro and in vivo, we report that exposure to insulin analogues was associated with the down-regulation of OPG, an inhibitor of VC. Furthermore, exposure to insulin glargine was associated with increased osteoblastic activity in HASMCs. Liraglutide, however, was not seen to exert any significant effects on OPG release, osteoblastic activity in HASMCs or VC within the coronary arteries.

Only two studies have previously examined the relationship between insulin therapy and OPG in the clinical setting. With regards to patients with type 2 diabetes, Jorgensen et al. (7) performed euglycemichyperglycemic clamp studies of $4 \mathrm{~h}$ duration, during which they examined the acute response of circulating OPG concentrations to the administration of insulin. They reported a significant decrease in circulating concentrations of the glycoprotein. Xiang et al. (16), in contrast, measured OPG levels at the onset of type 1 diabetes, and they reported that the commencement of insulin therapy was associated with a significant reduction in circulating concentrations of OPG over 6 months. The present study is the first to perform a long-term analysis of this relationship in patients with type 2 diabetes, and its results indicate that the down-regulation of OPG by insulin therapy is a significant and persistent effect at 16 months following the commencement of therapy. With regards to our in vitro data, we noted a highly significant reduction in OPG release from HASMCs following exposure to insulin glargine. Oleson et al. (9) previously reported a $70 \%$ reduction in the concentration of OPG in lysates of VSMCs exposed to human insulin. It is interesting, in this context, that the present data focused on OPG release into the media surrounding HASMCs as opposed to lysates, and in doing so, they demonstrated a much greater down-regulation of OPG. These findings suggest that in addition to decreasing the production of OPG by HASMCs, insulin exposure may also have significant effects on the release of this protein from cells.

OPG knock-out mice have been shown to demonstrate extensive and severe VC at an early age (5). Furthermore, the administration of OPG has been shown to decrease VC in atherogenic mice and to prevent vitamin D-induced VC in rodent models $(17,18)$. The precise mechanism by which OPG exerts these effects is the subject of ongoing debate, but recent findings have suggested that its inhibitory effects on VC may be mediated by the ability of OPG to block the actions of receptor activator of nuclear factor kappa- $\beta$ ligand (RANKL), another protein that has 


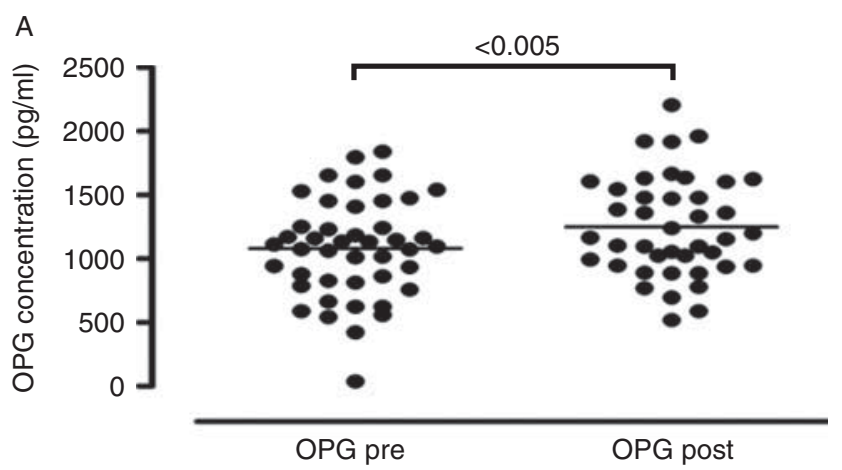

B
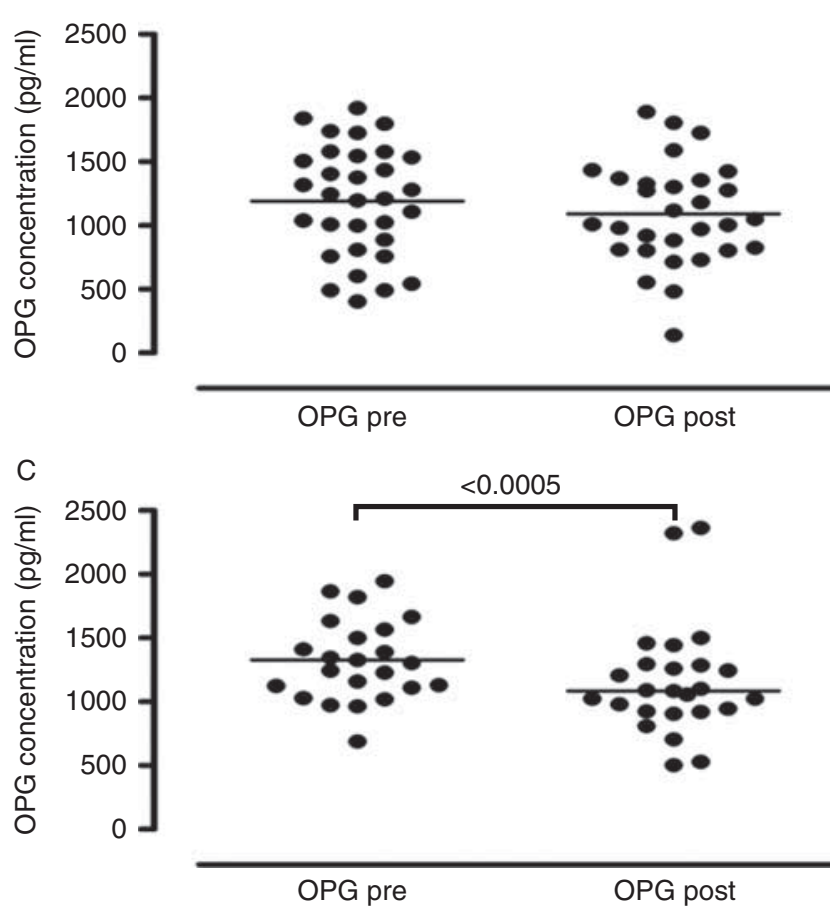

Figure 3

Circulating OPG concentrations as measured at baseline (pre) and at 16 months (post), stratified into the control group (A), the patients exposed to liraglutide (B) and the patients exposed to insulin analogues (C). Datapoints are provided, with lines indicating mean or median values where appropriate.

been identified within the vascular wall and one that has been reported to induce osteoblastic behaviour in VSMCs (19). Thus, the significant insulin-induced downregulation of OPG reported in the present paper may lead to unopposed RANKL activity, which gives rise to a proosteoblastic phenotype within the vasculature. This represents a potential explanation for the increased osteoblastic activity noted in the present study. To the best of our knowledge, no study has previously investigated the long-term effects of liraglutide on OPG concentrations in human subjects, and the present data suggest that this medication does not exert any significant effect on circulating concentrations of OPG.

With regards to $\mathrm{VC}$, although exposure to insulin analogue therapy was not associated with a significant increase in CAC burden when all baseline differences were taken into account, we nonetheless observed a trend towards an acceleration of VC in the insulin group. This trend is notable because it occurred in conjunction with a significant down-regulation of OPG, a known inhibitor of VC, and it parallels our in vitro data, in which insulin glargine promoted calcifying activity. Overall, these
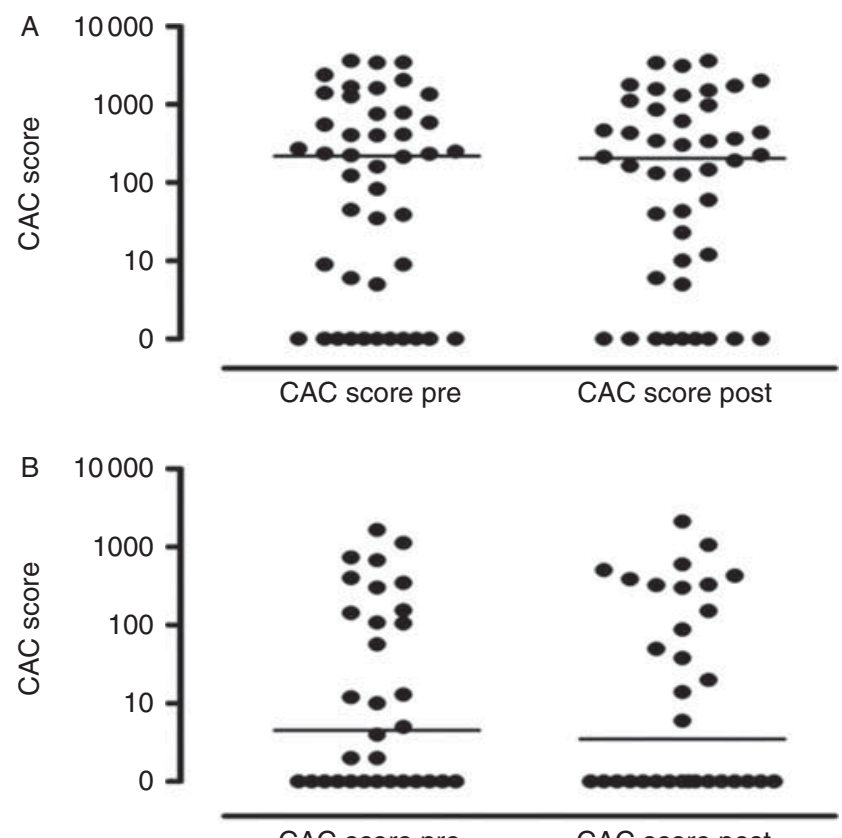

CAC score pre

CAC score post

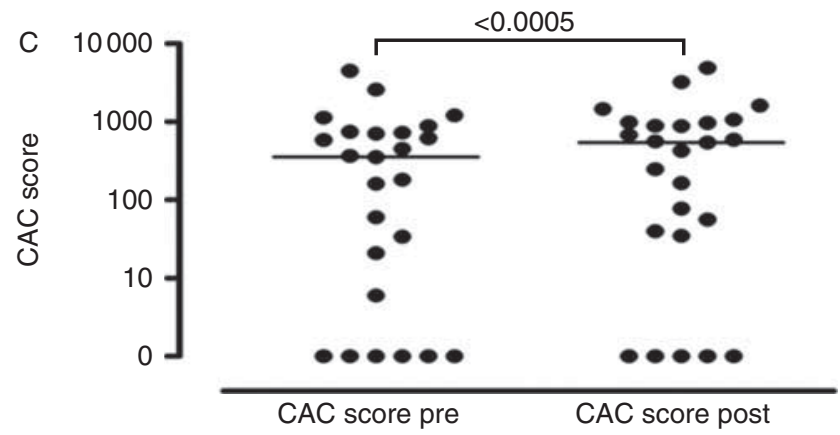

Figure 4

CAC scores (log) as measured by CT at baseline (pre) and at 16 months (post), stratified into the control group (A), the patients exposed to liraglutide (B) and the patients exposed to insulin analogues (C). Datapoints are provided, with lines indicating mean or median values where appropriate. 
results raise the possibility that insulin may promote VC, whereas no effect was seen with liraglutide at the cellular or systemic levels. Ultimately, therefore, we submit that randomised control trials are merited to further investigate this relationship. Although it would have been optimal to include such an approach in the present study, this research was the first to investigate the effects of these medications on VC in the clinical setting. As such, we felt it was both logistically and ethically necessary to adopt the present observational approach before randomised studies are established. Finally, we also note that the relationship between insulin therapy and VC is further complicated by additional effects of insulin on the vasculature: the reduction of hyperglycaemia in patients with type 2 diabetes leads to decreased oxidative stress, which is a recognised risk factor for VC (20). It is possible, therefore, that any calcifying effects of insulin therapy in the present study may have been balanced by the additional beneficial or 'pleiotropic' vascular effects of this medication in the complex in vivo setting. This explanation would be in keeping with the findings of clinical trials such as ORIGIN, which did not demonstrate increased macrovascular risk with insulin glargine (21). Once again, it is likely that randomised trials will be necessary to separate these effects.

There are a number of additional considerations regarding the present study. Although the magnitude of the reduction in OPG release from HASMCs exposed to insulin glargine raises the possibility of insulin interfering with the OPG ELISA, we tested this effect by exposing endothelial cell populations to similar insulin doses, and no reduction in OPG was observed, despite the presence of insulin glargine. Furthermore, we note that insulin glargine is capable of binding to and activating the insulin-like growth factor 1 (IGF1) receptor, a signalling system whose relevance to $\mathrm{VC}$ is unclear at present, to a greater degree than human insulin is (22). As such, it is possible that the osteoblastic effects noted with glargine occurred secondary to the activation of the IGF1 pathway and not via classic insulin signalling systems. It is also noteworthy that we did not utilise the deposition of calcium within the cell cultures as an endpoint in our in vitro experiments. Although this approach has been used by other groups to demonstrate the emergence of calcification, it requires the use of hyperphosphataemic 'calcifying' culture media, which introduces confounders into the experimental model, because phosphate itself induces osteoblastic change in VSMCs (23). By measuring $A L P$ activity and osteogenic gene expression in the absence of calcifying media, we were able to monitor the activity of a critical promoter of calcium crystal formation along with alterations of VSMC function towards an osteoblastic state without introducing additional confounders.

In conclusion, the present study represents the first investigation of the relationship between two widely utilized medications for the highly prevalent condition that is type 2 diabetes and the phenomenon of $\mathrm{VC}$, a condition that is increasingly being recognised as exerting a variety of deleterious effects on the CV system. Our data indicate that liraglutide does not affect this system either at the cellular or the systemic level. In contrast, insulin analogues were associated with a significant downregulation of OPG in vitro and in vivo, whereas osteoblastic activity was increased in HASMCs that were exposed to insulin. CAC was observed to increase in patients exposed to insulin, and although this change was potentially attributable to baseline differences, when considered as a whole, these results support the establishment of randomised studies into diabetes medications and VC in type 2 diabetes.

\section{Declaration of interest}

The authors declare that there is no conflict of interest that could be perceived as prejudicing the impartiality of the research reported.

\section{Funding}

This research was supported by a research training fellowship grant supplied by the Health Research Board of Ireland to CD (grant number HPF/2010/30).

Author contribution statement

C Davenport, F McGrath, B McAdam, P M Cummins and D Smith all contributed substantially to the study conception and design. C Davenport, W A Mahmood and $\mathrm{H}$ Forde contributed to the acquisition of data. D T Ashley contributed to the statistical analysis. A Agha, J McDermott, S Sreenan, C J Thompson, C Davenport, P M Cummins and D Smith contributed to the analysis and interpretation of the data. C Davenport, P M Cummins and D Smith drafted the article, and all authors revised it critically. CDavenport is responsible for the integrity of the work as a whole.

\section{References}

1 Johnson RC, Leopold JA \& Loscalzo J. Vascular calcification: pathobiological mechanisms and clinical implications. Circulation Research 200699 1044-1059. (doi:10.1161/01.RES.0000249379.55535.21)

2 Abedin M, Tintut Y \& Demer LL. Vascular calcification: mechanisms and clinical ramifications. Arteriosclerosis, Thrombosis, and Vascular Biology 200424 1161-1170. (doi:10.1161/01.ATV.0000133194. 94939.42) 
3 Schurgin S, Rich S \& Mazzone T. Increased prevalence of significant coronary artery calcification in patients with diabetes. Diabetes Care 200124 335-338. (doi:10.2337/diacare.24.2.335)

4 Chen NX \& Moe SM. Vascular calcification: pathophysiology and risk factors. Current Hypertension Reports 201214 228-237. (doi:10.1007/ s11906-012-0265-8)

5 Bucay N, Sarosi I, Dunstan CR, Morony S, Tarpley J, Capparelli C, Scully S, Tan HL, Xu W, Lacey DL et al. Osteoprotegerin-deficient mice develop early onset osteoporosis and arterial calcification. Genes and Development 199812 1260-1268. (doi:10.1101/gad.12.9.1260)

6 Tintut Y \& Demer L. Role of osteoprotegerin and its ligands and competing receptors in atherosclerotic calcification. Journal of Investigative Medicine 200654 395-401. (doi:10.2310/6650.2006.06019)

7 Jorgensen GM, Vind B, Nybo M, Rasmussen LM \& Hojlund K. Acute hyperinsulinemia decreases plasma osteoprotegerin with diminished effect in type 2 diabetes and obesity. European Journal of Endocrinology 2009161 95-101. (doi:10.1530/EJE-09-0141)

8 Nuche-Berenguer B, Moreno P, Esbrit P, Dapía S, Caeiro JR, Cancelas J, Haro-Mora JJ \& Villanueva-Peñacarrillo ML. Effect of GLP-1 treatment on bone turnover in normal, type 2 diabetic, and insulin-resistant states. Calcified Tissue International 200984 453-461. (doi:10.1007/ s00223-009-9220-3)

9 Olesen P, Nguyen K, Wogensen L, Ledet T \& Rasmussen LM. Calcification of human vascular smooth muscle cells: associations with osteoprotegerin expression and acceleration by high-dose insulin. American Journal of Physiology. Heart and Circulatory Physiology 2007292 H1058-H1064. (doi:10.1152/ajpheart.00047.2006)

10 Yuan LQ, Zhu JH, Wang HW, Liang QH, Xie H, Wu XP, Zhou H, Cui RR, Sheng ZF, Zhou HD et al. RANKL is a downstream mediator for insulininduced osteoblastic differentiation of vascular smooth muscle cells. PLoS ONE 20116 e29037. (doi:10.1371/journal.pone.0029037)

11 Pun KK, Lau P \& Ho PW. The characterization, regulation, and function of insulin receptors on osteoblast-like clonal osteosarcoma cell line. Journal of Bone and Mineral Research 19894 853-862. (doi:10.1002/jbmr. $5650040610)$

12 Wang CC, Sorribas V, Sharma G, Levi M \& Draznin B. Insulin attenuates vascular smooth muscle calcification but increases vascular smooth muscle cell phosphate transport. Atherosclerosis 2007195 e65-e75. (doi:10.1016/j.atherosclerosis.2007.02.032)

13 Costa CH, Batista MC, Moises VA, Kohlmann NB, Ribeiro AB \& Zanella MT. Serum insulin levels, 24-hour blood pressure profile, and left ventricular mass in nonobese hypertensive patients. Hypertension 199526 1085-1088. (doi:10.1161/01.HYP.26.6.1085)

14 Buse JB, Rosenstock J, Sesti G, Schmidt WE, Montanya E, Brett JH, Zychma M, Blonde L \& LEAD-6 Study Group. Liraglutide once a day versus exenatide twice a day for type 2 diabetes: a 26-week randomised, parallel-group, multinational, open-label trial (LEAD-6). Lancet 2009 374 39-47. (doi:10.1016/S0140-6736(09)60659-0)

15 Agatston AS, Janowitz WR, Hildner FJ, Zusmer NR, Viamonte M Jr \& Detrano R. Quantification of coronary artery calcium using ultrafast computed tomography. Journal of the American College of Cardiology 199015 827-832. (doi:10.1016/0735-1097(90)90282-T)

16 Xiang GD, Sun HL \& Zhao LS. Changes of osteoprotegerin before and after insulin therapy in type 1 diabetic patients. Diabetes Research and Clinical Practice 200776 199-206. (doi:10.1016/j.diabres.2006.09.008)

17 Morony S, Tintut Y, Zhang Z, Cattley RC, Van G, Dwyer D, Stolina M, Kostenuik PJ \& Demer LL. Osteoprotegerin inhibits vascular calcification without affecting atherosclerosis in $\operatorname{ld} \operatorname{lr}(-/-)$ mice. Circulation 2008117 411-420. (doi:10.1161/CIRCULATIONAHA.107.707380)

18 Price PA, June HH, Buckley JR \& Williamson MK. Osteoprotegerin inhibits artery calcification induced by warfarin and by vitamin $\mathrm{D}$. Arteriosclerosis, Thrombosis, and Vascular Biology 200121 1610-1616. (doi:10.1161/hq1001.097102)

19 Ndip A, Wilkinson FL, Jude EB, Boulton AJ \& Alexander MY. RANKL-OPG and RAGE modulation in vascular calcification and diabetes: novel targets for therapy. Diabetologia 201457 2251-2260. (doi:10.1007/s00125-014-3348-z)

20 Demer LL \& Tintut Y. Vascular calcification: pathobiology of a multifaceted disease. Circulation 2008117 2938-2948. (doi:10.1161/ CIRCULATIONAHA.107.743161)

21 ORIGIN Trial Investigators, Gerstein HC, Bosch J, Dagenais GR, Díaz R, Jung H, Maggioni AP, Pogue J, Probstfield J, Ramachandran A et al. Basal insulin and cardiovascular and other outcomes in dysglycemia. New England Journal of Medicine 2012367 319-328. (doi:10.1056/ NEJMoa1203858)

22 Bolli GB, Hahn AD, Schmidt R, Eisenblaetter T, Dahmen R, Heise T \& Becker RH. Plasma exposure to insulin glargine and its metabolites M1 and M2 after subcutaneous injection of therapeutic and supratherapeutic doses of glargine in subjects with type 1 diabetes. Diabetes Care 201235 2626-2630. (doi:10.2337/dc12-0270)

23 Cozzolino M, Gallieni M \& Brancaccio D. The mechanisms of hyperphosphatemia-induced vascular calcification. International Journal of Artificial Organs 200831 1002-1003.

Received 21 December 2014

Revised version received 11 March 2015

Accepted 13 April 2015 